\title{
The 2MASS Wide-Field T Dwarf Search. V. Discovery of a T Dwarf via Methane Imaging
}

\author{
S. C. Ellis and C. G. Tinney \\ Anglo-Australian Observatory, P.O. Box 296, Epping, NSW 2121, Australia \\ sce@aao.gov.au \\ cgt@aao.gov.au \\ Adam J. Burgasser ${ }^{1}$ \\ Department of Astrophysics, Division of Physical Sciences, American Museum of Natural History, Central \\ Park West at 79th Street, New York, NY 10024, USA \\ adam@amnh.org \\ J. Davy Kirkpatrick \\ Infrared Processing \& Analysis Center, Caltech, Pasadena, CA 91125, USA \\ davy@ipac.caltech.edu \\ and \\ Michael W. McElwain \\ UCLA, 8371 Mathematical Sciences, CA 90095, USA \\ mcelwain@astro.ucla.edu
}

\begin{abstract}
We present the discovery of a T dwarf, 2M2151-4853, via differential imaging through methane filters. The filters are designed to highlight the strong absorption in the $\mathrm{H}$ band, due to methane found in the atmospheres of $\mathrm{T}$ dwarfs, and provide a very efficient means of searching for them. Subsequent $\mathrm{J}$ and $\mathrm{H}$ band spectroscopy confirms 2M2151-4853 as a $\mathrm{T}$ dwarf of type T4.5. It has an estimated spectrophotometric distance of $18 \pm 3 \mathrm{pc}$, and an estimated tangential velocity of $v_{\mathrm{t}}=50 \pm 10 \mathrm{~km} \mathrm{~s}^{-1}$.
\end{abstract}

Subject headings: stars: individual: 2MASS J21513839-4853542 — stars: low-mass, brown dwarfs

\section{INTRODUCTION}

$\mathrm{T}$ dwarfs are the coolest $(T \lesssim 1500 \mathrm{~K}$, Vrba et al. 2004; Golimowski et al. 2004) and least luminous brown dwarfs observed. With atmospheres rich in molecular gases and condensate clouds (e.g. Ackerman \& Marley 2001), these sources represent a class of object intermediate between giant planets and low mass stars.

\footnotetext{
${ }^{1}$ Spitzer Fellow
}

In the cool atmospheres of $\mathrm{T}$ dwarfs, methane and water vapor are formed in abundance. These give rise to broad absorption features in the nearinfrared, which distinguish $\mathrm{T}$ dwarfs from hotter brown dwarfs in which the production of atmospheric methane is prohibited by collisional dissociation (Noll et al. 2000). These prominent $\mathrm{CH}_{4}$ and $\mathrm{H}_{2} \mathrm{O}$ features have been used to construct schemes of spectral classification for these objects (Burgasser et al. 2002; Geballe et al. 2002). Because of their low photospheric temperatures, 
an understanding the atmospheres of $\mathrm{T}$ dwarfs is likely to yield important clues about the nature of giant planetary atmospheres.

A statistically complete census of $\mathrm{T}$ dwarfs is highly desirable in an effort to extend our understanding of the initial mass function to lower masses, but cataloguing $\mathrm{T}$ dwarfs can be a time consuming process. With faint intrinsic magnitudes and near-infrared colors similar to most main sequence stars, $\mathrm{T}$ dwarf candidates can be buried in an overwhelming number of background sources. Traditional spectroscopic follow-up of large samples is extremely time consuming and has typically had low rates of success. Tinney et al. (2005) describe a new method for simplifying the search process, based on imaging through $\mathrm{CH}_{4}$ filters. Here we describe a new $\mathrm{T}$ dwarf discovered by this method.

Section 2 summarizes the project and the methane imaging process. Section 3 describes the observations and empirical properties of the new T dwarf 2MASS J21513839-4853542, hereafter 2M2151-4853. Conclusions are given in Section 4 .

\section{THE 2MASS WIDE-FIELD T DWARF SEARCH AND METHANE IMAG- ING}

The 2MASS Wide-Field T Dwarf Search is an attempt to conduct a census of the T dwarf population over most of the sky. The selection process is described in detail by Burgasser et al. (2003b), and is summarized here.

The initial sample has been culled from the 2MASS All Sky Working Database (Skrutskie et al. 1997; Cutri et al. 2003), via color and magnitude selection. Areas within a galactic latitude $|b|<15^{\circ}$ have been excluded to avoid regions where source confusion will be problematic. Likewise the Magellanic clouds, and other dense source regions have been omitted. To facilitate follow up observations, regions within $2^{\circ}$ of the equatorial poles were also omitted. In total the search covers $74 \%$ of the sky.

From this sample objects were selected with $J-H \leq 0.3$ or $H-K_{\mathrm{S}} \leq 0, J \leq 16$, and no counterpart in the USNO-A2.0 catalogue (Monet et al. 1998), effectively $R-J>4$. This range of color is sufficiently broad to accommodate warmer
T dwarfs, as early as type T1 (Tinney et al. 2005).

This selection process yields over 250,000 targets. Since it is expected that there will only be 20-30 T dwarfs in this sample (Burgasser et al. 2003b), further discrimination of the targets has been made on the basis of visual classification by AJB and MWM, leaving $\sim 1500$ targets. Targets from this list are being observed as part of a methane imaging program.

Our methane imaging program of 2MASS $\mathrm{T}$ dwarf candidates is described in full by Tinney et al. (2005). Images were taken through the $\mathrm{CH}_{4} \mathrm{~S}$ and $\mathrm{CH}_{4} \mathrm{l}$ filters with the infrared camera and spectrograph (IRIS2) on the $3.9 \mathrm{~m}$ AngloAustralian Telescope, Siding Spring, Australia.

The $\mathrm{CH}_{4} \mathrm{l}$ filter covers the wavelength range of the strong methane absorption features in the infrared $\mathrm{H}$ band, which define the spectral type $\mathrm{T}$ dwarfs (Geballe et al. 2002). Meanwhile the $\mathrm{CH}_{4} \mathrm{~s}$ filter samples a wavelength range outside the methane absorption (though within the range of absorption by other molecules such as $\mathrm{H}_{2} \mathrm{O}$ ).

The methane observations were calibrated via a series of extensive observations described in Tinney et al. (2005). Stars of known spectral standard were observed, and then calibrated according to observations of $\mathrm{A}, \mathrm{F}$ and $\mathrm{G}$ stars which show virtually no color variation in $\mathrm{CH}_{4} \mathrm{~s}-\mathrm{CH}_{4} \mathrm{l}$, and $\mathrm{K}$ and $\mathrm{M}$ stars for which the methane colors have been empirically calibrated.

\section{OBSERVATIONS}

\subsection{Methane Imaging}

Methane observations of 2M2151-4853, as described above, were made on 2003 June 11 (UT), in patchy cloud (which appears not to have affected our imaging) and 1.1 arcsec seeing. Exposures of 30 seconds in each of the $\mathrm{CH}_{4} \mathrm{~S}$ and $\mathrm{CH}_{4} \mathrm{l}$ filters were made. Images were dark subtracted and flat fielded using standard procedures with the pipeline reduction software, ORAC-DR. The flat fields were made by median combining jittered images of the targets. Finding charts extracted from these images and the 2MASS $\mathrm{H}$ band image are shown in Figure 1. SExtRACTOR (Bertin \& Arnouts 1996) was used to measure magnitudes in fixed apertures of $\approx 2.5$ arcsec diameter. The differential photometry was calibrated using the 
procedure described in Tinney et al. (2005) using 2MASS sources in the field, to produce Figure 2.

A plot of $\mathrm{CH}_{4} \mathrm{~S}-\mathrm{CH}_{4} \mathrm{l}$ against $\mathrm{CH}_{4} \mathrm{~S}$ is shown in Figure 2 with 2M2151-4853 clearly visible above the other objects in the field. A comparison of the methane color of 2M2151-4853 with the calibrated methane colors of A stars through to $\mathrm{T}$ dwarfs is shown in Figure 3, taken from Tinney et al. (2005). The color of $\mathrm{CH}_{4} \mathrm{~s}-\mathrm{CH}_{4} \mathrm{l}=-0.15 \pm 0.03$ suggests that 2M2151-4853 is of type T2.5 \pm 1 . Figure 3 highlights the power of the methane imaging methodology. Short images of the target field are made through the $\mathrm{CH}_{4}$ filters. Any $\mathrm{T}$ dwarfs present in the field will be immediately obvious in a plot of $\mathrm{CH}_{4} \mathrm{~s}-\mathrm{CH}_{4} \mathrm{l}$, standing out prominently from the locus of points made up from the hotter stars. Furthermore, a first estimate of spectral type within the $\mathrm{T}$ dwarf class can be made from the methane color. Thus only $\mathrm{T}$ dwarf candidates with unusual methane colors ever need to be followed up with spectroscopic observations.

\subsection{Spectroscopy}

Hs band spectroscopy of 2M2151-4853 was obtained using IRIS2 on 2004 June 23 (UT). The seeing was $\sim 1.1$ arcseconds, and there was patchy cloud cover. This spectrum was taken with very low signal to noise, sufficient to identify 2M21514853 as a $\mathrm{T}$ dwarf, but not to classify its spectral type. Higher signal to noise spectra spectra were then obtained on 2004 August 22 (UT) under good conditions (seeing $\sim 1.2$ arcseconds and clear sky) in the $\mathrm{Hs}$ and $\mathrm{Jl}$ bands. The total exposure was 15 minutes in each band. The spectra were flat-fielded using dome flats created from a lamp-on-lamp-off sequence. The wavelength calibration was achieved through imaging of a Xe arc lamp. The flattened spectra were divided by a normalized spectrum of a $\mathrm{G}$ dwarf telluric standard star to remove the effect of absorption bands.

The final spectra, binned by a factor of six to improve signal to noise, are shown in Figure 4, along with the spectrum of the T4.5 dwarf, 2MASS J05591914-1404488, hereafter 2M05591404 (Burgasser et al. 2000; Tinney et al. 2005). The distinctive absorption features due to $\mathrm{H}_{2} \mathrm{O}$ and $\mathrm{CH}_{4}$ are clearly visible, and are identified at the top of each figure, along with the KI absorption lines in the $\mathrm{Jl}$ band.
Spectral classification schemes have been devised by Burgasser et al. (2002) and Geballe et al. (2002). We have used the spectral indices of both schemes within the wavelength range of our spectra, to check the classification based on the methane colors described above.

Both schemes define spectral indices based on distinctive $\mathrm{H}_{2} \mathrm{O}$ and $\mathrm{CH}_{4}$ features in T dwarf spectra. Burgasser et al. (2002) define the diagnostics given in equation 1 , based on the mean flux within the regions of interest, and those of a neighboring region of the spectrum outside the absorption feature, and calculating their ratio. For the case of 2M2151-4853 we have,

$$
\begin{array}{r}
\mathrm{H}_{2} \mathrm{O}-A=\frac{\left\langle F_{1.12-1.17}\right\rangle}{<F_{1.25-1.28}>}=0.37 \\
\mathrm{H}_{2} \mathrm{O}-B=\frac{\left.<F_{1.505-1.525}\right\rangle}{<F_{1.575-1.595}>}=0.46 \\
\mathrm{CH}_{4}-A=\frac{\left.<F_{1.295-1.325}\right\rangle}{<F_{1.25-1.28}>}=0.81 \\
\mathrm{CH}_{4}-B=\frac{\left.<F_{1.640-1.700}\right\rangle}{\left.<F_{1.575-1.595}\right\rangle}=0.49,
\end{array}
$$

where the subscripts denote the wavebands of interest in $\mu \mathrm{m}$. Burgasser et al. (2002) list index values for $\mathrm{T}$ dwarf standards in their table 10 . A comparison with that table shows that 2M21514853 lies between type T3 and T5 for all the diagnostics (there is no type T4 standard listed).

Geballe et al. (2002) define similar indices based on the integrated flux from diagnostic regions of $\mathrm{T}$ dwarf spectra. Computing their indices for 2M2151-4853 we find,

$$
\begin{aligned}
& \mathrm{CH}_{4} \text { index }=\frac{\int_{1.56}^{1.60} f_{\lambda} \mathrm{d} \lambda}{\int_{1.635}^{1.675} f_{\lambda} \mathrm{d} \lambda}=1.82 \\
& \mathrm{H}_{2} \text { Oindex }=\frac{\int_{1.26}^{1.29} f_{\lambda} \mathrm{d} \lambda}{\int_{1.13}^{1.16} f_{\lambda} \mathrm{d} \lambda}=2.66,
\end{aligned}
$$

where the limits of the integrals are given in $\mu \mathrm{m}$. Geballe et al. (2002) present a look-up table of indices for different spectral types. Comparing to this (table 5 in their paper), we find that the $\mathrm{CH}_{4}$ and $\mathrm{H}_{2} \mathrm{O}$ indices indicate that 2M2151-4853 is of type $\mathrm{T} 5$ and $\mathrm{T} 4$ respectively.

A hybrid system between the schemes of Burgasser et al. (2002) and Geballe et al. (2002) is in 

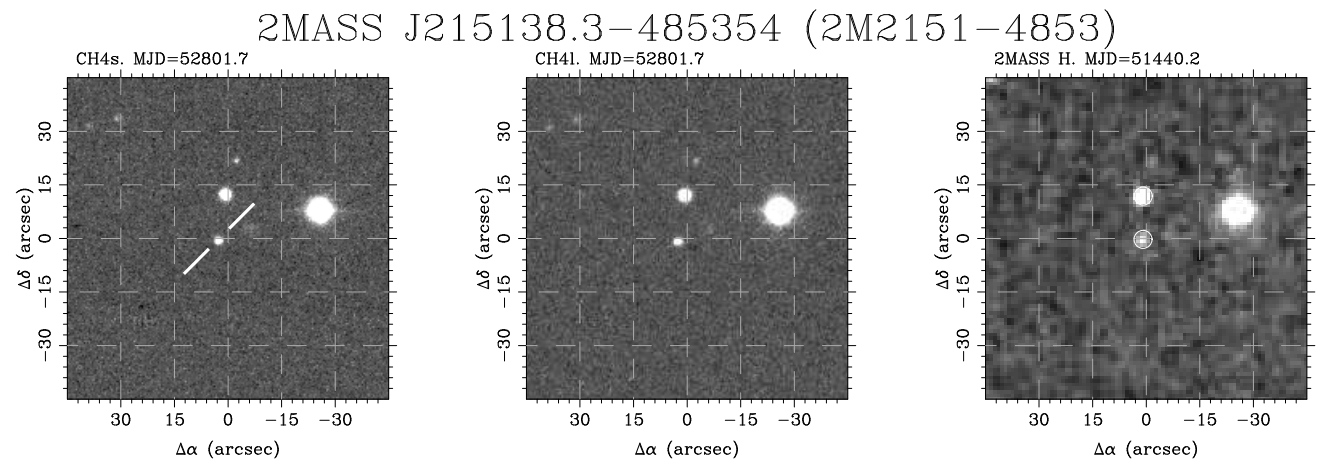

Fig. 1.- Finding chart for 2M2151-4853. The images are 1.5 arcminutes on a side, and are centered on the 2MASS coordinates, $21^{\mathrm{h}} 51^{\mathrm{m}} 38.39^{\mathrm{s}},-48^{\circ} 53^{\prime} 54.2^{\prime \prime}$, and oriented with North up and East toward the left. The slight offset from the 2MASS coordinates illustrates the substantial proper motion of 2M2151-4853 of $0.57 \pm 0.07$ arcseconds year $^{-1}$, see Section 3.4.

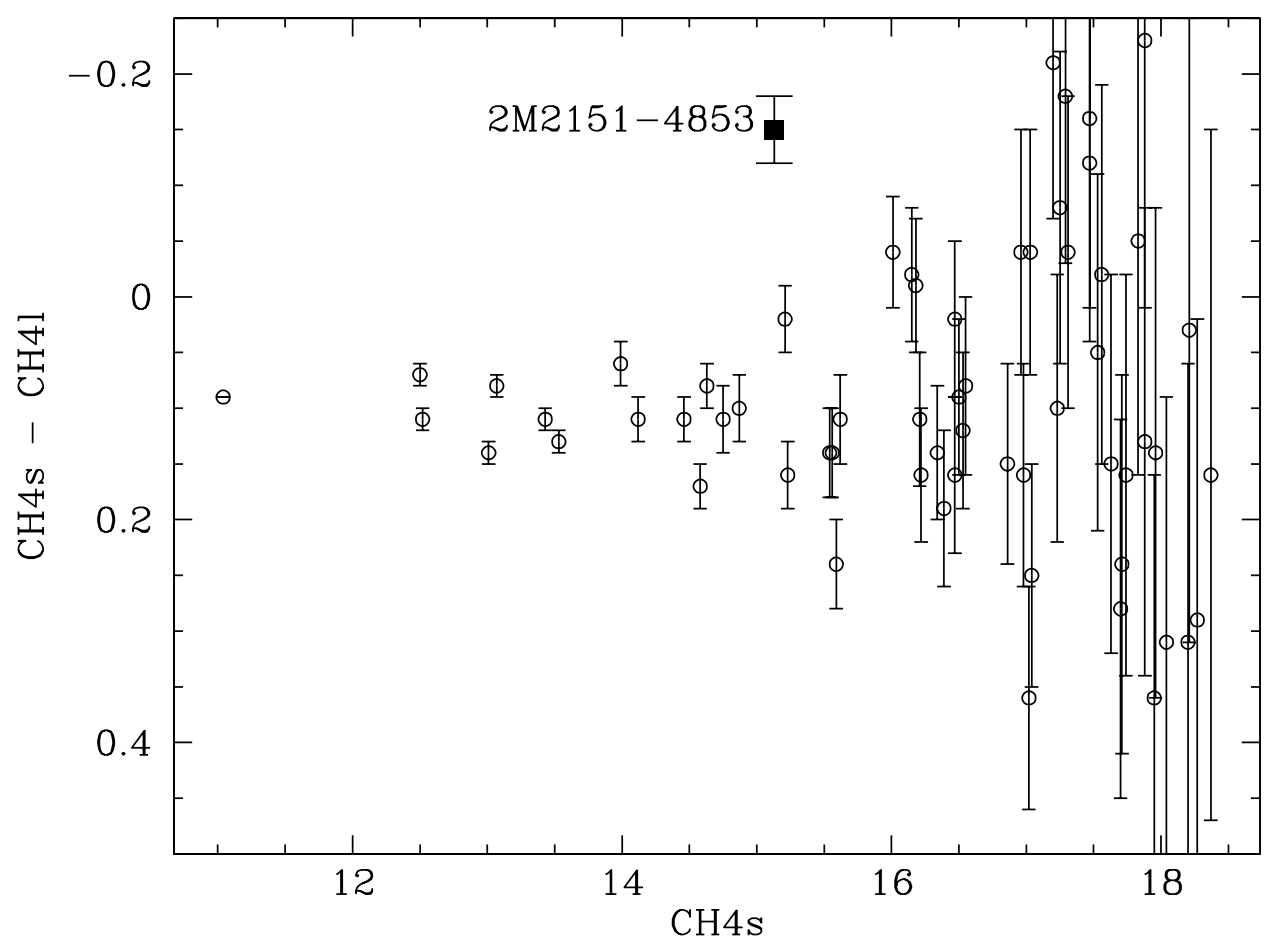

Fig. 2. $-\mathrm{CH}_{4} \mathrm{~s}-\mathrm{CH}_{4} \mathrm{l}$ vs. $\mathrm{CH}_{4} \mathrm{~s}$ color-magnitude diagram. 2M2151-4853 is plotted as the filled square and is obvious at $\mathrm{CH}_{4} \mathrm{~s}=15.13$ by its excess methane color. 


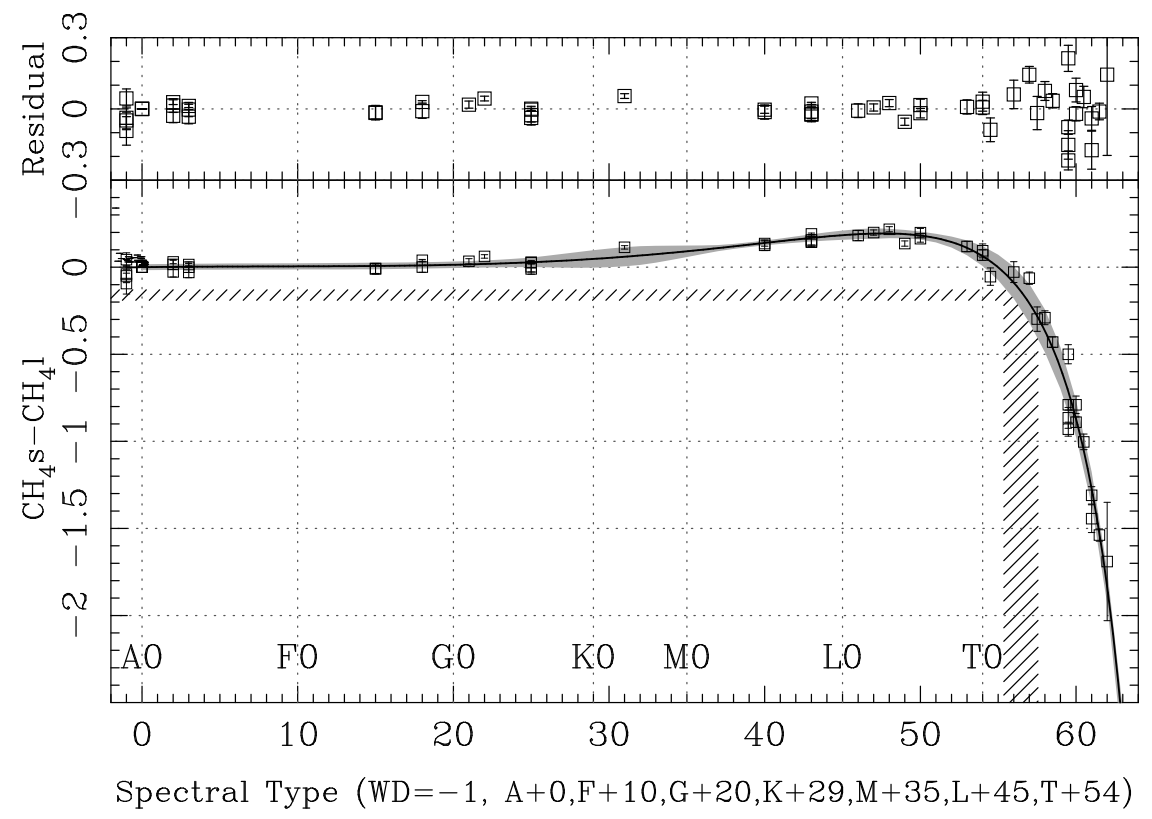

Fig. 3.- Methane color as a function of spectral-type. The shaded region around the curve represents the uncertainties derived from photon counting statistics, aperture correction uncertainties and photometric calibration uncertainties. The horizontal and vertical hatched region represents the methane color and uncertainty of 2M2151-4853 and the corresponding spectral type. See Tinney et al. (2005) for full details.
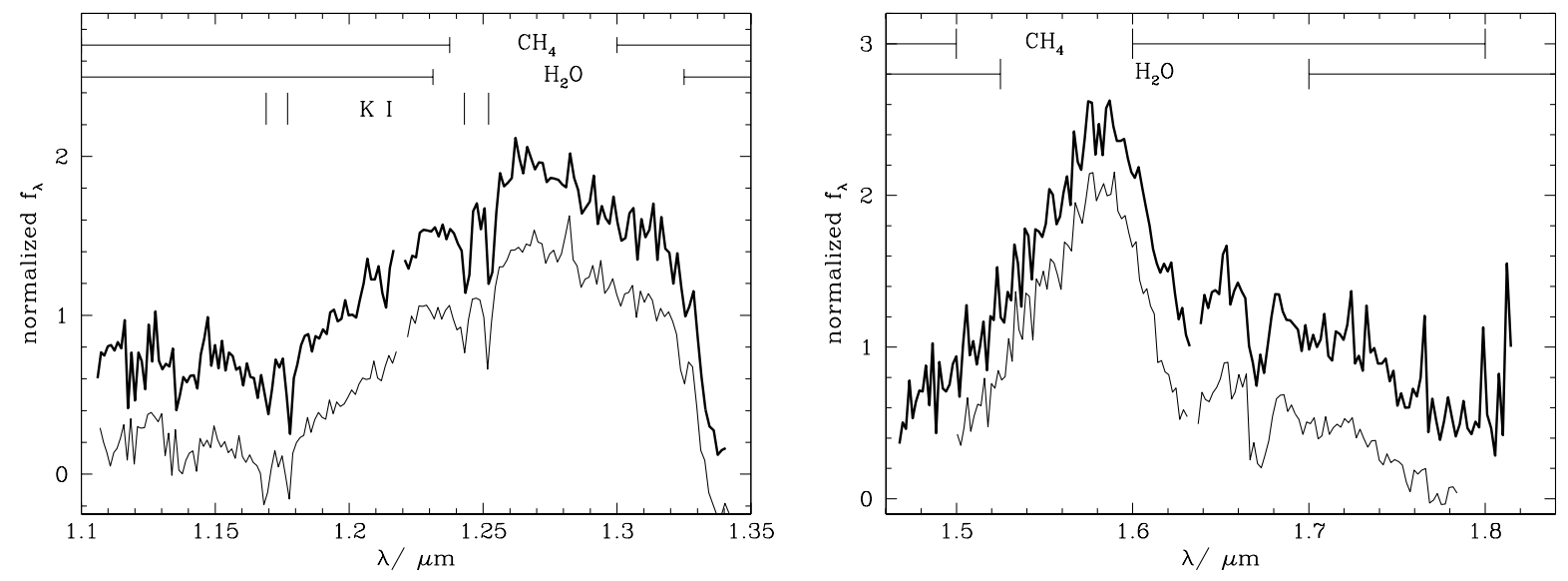

Fig. 4. - The Jl (left panel) and Hs (right panel) band spectra of 2M2151-4853 are shown by the upper bold lines. The lower spectra are those of the T4.5 dwarf, 2M0559-1404, and are offset by a constant of 0.5 for clarity. The $\mathrm{Jl}$ band spectra have been normalized at $\lambda=1.2 \mu \mathrm{m}$ and the Hs band spectra at $\lambda=1.7 \mu \mathrm{m}$. The $\mathrm{H}_{2} \mathrm{O}$ and $\mathrm{CH}_{4}$ absorption bands are indicated along with the KI absorption lines. 
development, utilizing a unified set of fundamental $\mathrm{T}$ dwarfs and spectroscopic indices (Burgasser et al. 2005). We compared a subset of the revised spectral indices for this scheme (presented in Burgasser et al. 2003a) between the spectrum of 2M2151-4853 and those of a set of T dwarf comparison sources (Tinney et al. 2005). These indices yield a mean spectral type of T4.5 for 2M21514853 , consistent with the above indices. This classification is further verified by the similarity of $\mathrm{J}$ and $\mathrm{H}$ band spectra with those of 2M0559-1404 (Figure 4), itself a T4.5 dwarf (Geballe et al. 2002).

\subsection{Spectrophotometric Distance}

The 2MASS photometry available for 2M21514853 has been used to derive an estimate of distance. We have compared the 2MASS magnitudes to the spectral type-absolute magnitude relations given by Tinney et al. (2003) and Vrba et al. (2004). In order to make the comparison with Vrba et al. (2004) the 2MASS magnitudes were first transformed to the CIT magnitude system using the relations of Stephens \& Leggett (2004). The results are given in Table 1.

The distances calculated from the relations of Tinney et al. (2003) and Vrba et al. (2004) are $21 \pm 2 \mathrm{pc}$ and $17 \pm 2 \mathrm{pc}$ respectively. The average distance using all relations is $18 \pm 3 \mathrm{pc}$ assuming it is single.

\subsection{Proper Motion}

2M2151-4853 has an appreciable proper motion measured at $0.57 \pm 0.07$ arcseconds year $^{-1}$ with a position angle of $113 \pm 3$ degrees, based on the difference in position of the 2MASS images and the methane images. Using the distance estimate derived above this translates to a tangential velocity of $v_{\mathrm{t}}=50 \pm 10 \mathrm{~km} \mathrm{~s}^{-1}$.

\section{CONCLUSIONS}

The discovery of 2M2151-4853 further emphasizes the power and efficiency of methane imaging in searching for $\mathrm{T}$ dwarfs. This method has been used to great success in the 2MASS widefield $\mathrm{T}$ dwarf search, as demonstrated in Tinney et al. (2005).

We have used the spectral classification schemes of Burgasser et al. (2002), Geballe et al. (2002) and
Burgasser et al. (2005) to classify 2M2151-4853. All schemes are consistent with 2M2151-4853 being of type T4.5. This is confirmed in a comparison of the spectra of 2M2151-4853 with those of the T4.5 dwarf, 2M0559-0414. Spectrophotometric distances indicate it is at a distance of $18 \pm 3 \mathrm{pc}$ and its proper motion implies a tangential velocity of $v_{\mathrm{t}}=50 \pm 10 \mathrm{~km} \mathrm{~s}^{-1}$, consistent with disk population kinematics. The properties of 2M2151-4853 are summarized in Table 2.

A total of $11 \mathrm{~T}$ dwarfs have now been identified in the 2MASS sample via methane imaging; however, our candidate sample is not yet exhausted. Further discoveries and a final accounting of the search will be provided in a forthcoming paper.

\section{Acknowledgments}

The authors wish to gratefully acknowledge the Joint Astronomy Centre, Hawaii, for making their ORAC-DR code available, and for assisting the AAO in implementing it for IRIS2. SCE wishes to acknowledge PPARC support whilst working on this paper. AJB acknowledges support by NASA through the SIRTF Fellowship Program.

This publication makes use of data from the Two Micron All Sky Survey which is a joint project of the University of Massachusetts and the Infrared Processing and Analysis Center, funded by NASA and the NSF.

2MASS data were obtained from the NASA/IPAC Infrared Science Archive, which is operated by the Jet Propulsion Laboratory, California Institue of Technology, under contract with the National Aeronautics and Space Administration.

\section{REFERENCES}

Ackerman, A. S., Marley, M. S. 2001, ApJ, 556, 872

Bertin, E., Arnouts, S. 1996, A\&AS, 117, 393

Burgasser, A. J., Geballe, T. R., Leggett, S. R., Kirkpatrick, J. D., Golimowski, D. A. 2005, ApJ, submitted

Burgasser, A. J., Golimowski, D. A., Geballe, T. R. ., Leggett, S. K., Kirkpatrick, J. D., Knapp, G. R., Fan, X. 2003a, in IAU Symposium 211: Brown Dwarfs, ed. E. L. Martín (San Francisco: ASP), 377 
Table 1: Photometry and derived distances

\begin{tabular}{llll}
\hline \hline & $\mathrm{J}$ & $\mathrm{H}$ & $\mathrm{K} / \mathrm{Ks}$ \\
\hline Apparent magnitude 2M2151(2MASS) & $15.73 \pm 0.08$ & $15.17 \pm 0.10$ & $15.43 \pm 0.18$ \\
Apparent magnitude 2M2151(CIT) & $15.55 \pm 0.08$ & $15.22 \pm 0.10$ & $15.57 \pm 0.19$ \\
Expected absolute magnitude (2MASS, Tinney et al. 2003) & $14.0 \pm 0.4$ & & $14.0 \pm 0.4$ \\
Expected absolute magnitude (CIT, Vrba et al. 2004) & 14.5 & 14.3 & 14.2 \\
Derived distance /pc (Tinney et al. (2003)) & 23 & 19 \\
Derived distance /pc (Vrba et al. 2004) & 16 & 15 & 19 \\
\hline
\end{tabular}

${ }^{a}$ Calculated from the near-infrared color transformations of Stephens \& Leggett (2004)

Table 2: Summary of the Properties of 2M2151-4853

\begin{tabular}{ll}
\hline \hline R.A. at 2003 June 11 (UT J2000.0) & $21^{\mathrm{h}} 51^{\mathrm{m}} 38^{\mathrm{s}} .6$ \\
Dec. at 2003 June 11 (UT J2000.0) & $-48^{\circ} 53^{\prime} 55^{\prime \prime}$ \\
2MASS J mag & $15.73 \pm 0.07$ \\
2MASS H mag & $15.17 \pm 0.10$ \\
2MASS Ks mag & $15.43 \pm 0.18$ \\
CH4s mag & $15.13 \pm 0.02$ \\
CH4l mag & $15.28 \pm 0.02$ \\
Proper motion & $0.57 \pm 0.07 \operatorname{arcsec~}^{-1}$ \\
Position angle & $113 \pm 3$ degrees \\
Distance & $18 \pm 3 \mathrm{pc}$ \\
Tangential velocity & $50 \pm 10 \mathrm{~km} \mathrm{~s}^{-1}$ \\
\hline
\end{tabular}


Burgasser, A. J., Kirkpatrick, J. D., McElwain, M. W., Cutri, R. M., Burgasser, A. J., Skrutskie, M. F. 2003b, AJ, 125, 850

Burgasser, A. J., et al. 2000, AJ, 120, 1100

Burgasser, A. J., et al. 2002, ApJ, 564, 421

Cutri, R., et al. 2003, Explanatory Supplement to the 2MASS All Sky Data Release, http://www.ipac.caltech.edu/2mass/releases/allsky/doc/explsup.html

Geballe, T. R., et al. 2002, ApJ, 564, 466

Golimowski, D. A., et al. 2004, AJ, 127, 3516

Monet, D. B. A., et al. 1998, VizieR Online Data Catalog, 1252

Noll, K. S., Geballe, T. R., Leggett, S. K., Marley, M. S. 2000, ApJ, 541, L75

Skrutskie, M. F., et al. 1997, in ASSL Vol. 210: The Impact of Large Scale Near-IR Sky Surveys, 25

Stephens, D. C., Leggett, S. K. 2004, PASP, 116, 9

Tinney, C. G., Burgasser, A. J., Kirkpatrick, J. D. 2003, AJ, 126, 975

Tinney, C. G., Burgasser, A. J., Kirkpatrick, J. D., McElwain, M. W. 2005, AJ, in press

Vrba, F. J., et al. 2004, AJ, 127, 2948

This 2-column preprint was prepared with the AAS LATEX macros v 5.2 . 